\title{
Molecular population divergence and sexual selection on morphology in the banded demoiselle (Calopteryx splendens)
}

\author{
EI Svensson ${ }^{1}$, L Kristoffersen ${ }^{2}, \mathrm{~K}$ Oskarsson ${ }^{1}$ and S Bensch ${ }^{1}$ \\ ${ }^{1}$ Section for Animal Ecology, Department of Ecology, Lund University, SE-223 62 Lund, Sweden; ${ }^{2}$ Section for Chemical Ecology and \\ Ecotoxicology, Department of Ecology, Lund University, SE-223 62 Lund, Sweden
}

\begin{abstract}
The importance of sexual selection in population divergence is of much interest, mainly because it is thought to cause reproductive isolation and hence could lead to speciation. Sexually selected traits have been hypothesized to diverge faster between populations than other traits, presumably because of differences in the strength, mechanism or dynamics of selection. We investigated this by quantifying population divergence in eight morphological characters in 12 south Swedish populations of a sexually dimorphic damselfly, the banded demoiselle (Calopteryx splendens). The morphological characters included a secondary sexual character, the male melanized wing spot, which has an important function in both inter- and intrasexual selection. In addition, we investigated molecular population divergence, revealed by amplified fragment length polymorphism (AFLP) analysis. Molecular population divergence was highly signi-
\end{abstract}

ficant among these Northern European populations (overall $F_{\mathrm{st}}=0.054$; pairwise population $F_{\mathrm{st}}$ 's ranged from $\sim 0$ to $0.13)$. We found evidence for isolation-by-distance $(r=0.70)$ for the molecular markers and a significant correlation between molecular and phenotypic population divergence $(r=0.39)$. One interpretation is that population divergence for the AFLP loci are affected by genetic drift, but is also indirectly influenced by selection, due to linkage with loci for the phenotypic traits. Field estimates of sexual and natural selection from two of the populations revealed fairly strong sexual selection on wing spot length, indicating that this trait has the potential to rapidly diverge, provided that variation is heritable and the observed selection is chronic.

Heredity (2004) 93, 423-433. doi:10.1038/sj.hdy.6800519

Published online 14 July 2004

Keywords: condition; gene flow; local adaptation; mate preferences; melanin; runaway process

\section{Introduction}

Phenotypic evolution is often viewed as a process in which populations become adapted to their local environments under the influence of natural and/or sexual selection (Charlesworth et al, 1982; Hansen, 1997; Schluter, 2000). According to this view, populations will quickly reach their local adaptive peaks, and selection plays a predominant role in population divergence (Lande, 1980). However, even if selection has a predominant influence on phenotypic evolution, nonadaptive processes could also be important. Genetic drift, founder events, gene flow and recombination may displace populations from their local adaptive peaks to a greater or lesser degree, depending on the relative balance between selection and these nonadaptive forces (Slatkin, 1985; Coyne et al, 1997; Wade and Goodnight, 1998; Fear and Price, 1998). In spite of these insights, studies of natural and sexual selection on phenotypic traits (eg Hoekstra et al, 2001) are typically performed in isolation from studies of genetic drift and molecular population divergence (Merilä and Crnokrak, 2001).

Correspondence: EI Svensson, Section for Animal Ecology, Department of Ecology, Lund University, SE-223 62 Lund, Sweden.

E-mail: erik.svensson@zooekol.lu.se

Received 22 October 2003; accepted 11 May 2004; published online 14 July 2004
Recently, Schluter (2000) reviewed the various empirical methods used to study adaptive population divergence, and he argued that traditional phenotypic experiments (eg reciprocal transplants) and molecular approaches (eg comparisons between morphological and molecular divergence) should preferably be integrated and combined in single studies.

There is currently much interest in the role of sexual selection in population divergence. While much previous work on sexual selection was directed towards correlates of male mating success within populations, there has been increased attention to the interpopulational consequences of sexual selection. A better understanding of the role of sexual selection in population divergence should also increase our knowledge about its role in speciation, since it is thought that reproductive isolation can evolve as a correlated response to sexual selection (Iwasa and Pomiankowski, 1995) or natural selection (Rice and Hostert, 1993), or through a combination of these forces.

Most workers seem to agree that secondary sexual traits and other traits involved in social interactions are evolving faster than nonsocial traits (West-Eberhard, 1983; Rice and Holland, 1997). This belief is based on the observation that closely related species (eg congeners) often differ more markedly in secondary sexual characters than in other characters (Darwin, 1871; Lande, 1981). The apparent arbitrary nature of the differences in secondary sexual characters between closely related taxa 
has been taken as an argument that speciation often results from runaway sexual selection (Fisher, 1930; Lande, 1981, 1982, 2000). However, the fact that closely related species often differ mostly in their secondary sexual characters is in itself no rigorous proof that sexual selection caused speciation, because the interspecific differences could have arisen after reproductive isolation was already accomplished, through a process of reproductive character displacement (Butlin, 1987, 1989). In spite of the widespread belief that sexually selected characters are evolving faster than nonsexually selected ones, there are still rather few quantitative studies of this at the intraspecific level (see Irwin et al (2001) for an exception). This is somewhat surprising, since studies of population variation in sexually selected traits have accumulated over the last years (Dale et al, 1999; Tregenza et al, 2000; Uy and Borgia, 2000; Panhuis et al, 2001).

In this paper, we present data on both molecular population divergence and population variation in secondary sexual characters of a calopterygid damselfly, the banded demoiselle Calopteryx splendens (Figure 1). The genus Calopteryx is interesting both from the viewpoint of population divergence and sexual selection. The large, dark wing colours and patches are used in both intra- and intersexual selection, and wing colouration among the North American species of this genus was subject to one of the first pioneering studies of reproductive character displacement (Waage, 1979). Studies on European species of the genus have revealed that the melanized wing patches are variable, both within and among populations (Askew, 1988). Colouration is also correlated with male disease resistance and immunological condition (Rantala et al, 2000; Siva-Jothy, 2000), giving the wing colour patches a potential role as

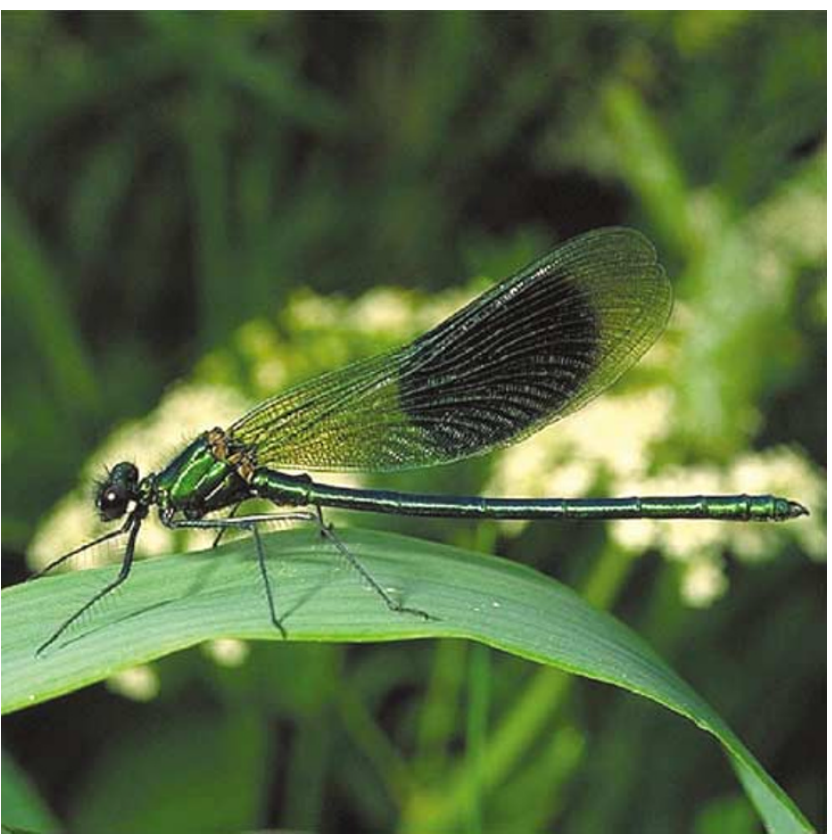

Figure 1 Male C. splendens. Note the large, melanized wing patches that have both inter- and intrasexual functions. Photograph was taken by Robert Thompson (Ireland) and reproduced with his permission. indicator traits in sexual selection through female choice (Andersson, 1994; Siva-Jothy, 1999). In this paper, we utilize both molecular and morphological data to get a better understanding of the causes of population divergence in morphology of C. splendens.

The banded demoiselle ( $C$. splendens) is a common insect along slowly running water streams in Europe, although it has decreased markedly during the last decades (Askew, 1988). C. splendens spends 1-4 years in the aquatic larval stage before emerging as adults, the length of the larval period increasing with increasing latitude (Sandhall, 2000). Emergence of adults takes place from late May and proceeds mainly through June in our study area in southern Sweden. Immediately after emergence, teneral individuals spend most of their time away from water as they become sexually mature and their exoskeleton hardens (Corbet, 1999). After sexual maturation, males and females return to water. Mating and oviposition take place exclusively at or near the water surface (Corbet, 1999). Males are polygynous and territorial and are involved in many combats with each other while trying to attract females and obtain matings (Plaistow and Siva-Jothy, 1996). Females usually oviposit soon after mating, and the number of copulations a male obtains during his reproductive life provides a good estimate of his lifetime reproductive success (Corbet, 1999). The territorial and sedentary habits of adult damselflies enables detailed studies of behaviour, survivorship and reproductive success, and consequently damselflies and other odonates have been subject to some previous studies of sexual and natural selection in the wild (eg Fincke, 1986; Koenig and Albano, 1987; Anholt, 1991; Grether, 1996a,b). Moreover, the discrete and largely isolated nature of the preferred aquatic habitat (rivers and streams) should make damselflies a particularly interesting group from the point of view of genetic population structure. Recently, there has been an accelerated interest in molecular population divergence, dispersal and gene flow in aquatic or semiaquatic invertebrates (eg Hughes et al, 1999; Miller et al, 2002; Schultheis et al, 2002; Bohonak and Jenkins, 2003). However, with the exception of a recent study using allozymes (Geenen et al, 2000), there are to our knowledge no previously published studies on gene flow or molecular population divergence in damselflies.

\section{Methods}

\section{Population survey, sampling and morphometrics}

We visited 12 south Swedish populations of C. splendens during June-August 2000. Distance between populations varied between 5 and $150 \mathrm{~km}$ (Supplementary Table 1). At each locality, we tried to catch and measure the morphology of at least 15 different males, although we were not able to achieve this goal for all populations (Supplementary Table 1). All recorded data were directly written into a portable handheld computer (Palm Pilot III), and upon return to our laboratory transferred onto a desktop PC. After morphological measurements and colour assessments (see below), we either released the males at their site of capture, or transferred some of them into vials containing a solution of $95 \%$ ethanol, with the specific aim of using them in later molecular analysis (see 'Molecular analyses' below). Each captured male 
was first classified by age, being either 'immature' or 'adult', depending on the stiffness of the wings (see Plaistow and Tsubaki (2000) for rationale). Individuals with paper-like, crisp wings and firm veins were classified as adults, whereas immatures have much softer, more pliant wings and a sheen reminiscent of cellofane. Body colour and wing spot colouration is also paler among immatures. The following morphological characters were measured in all males: thorax length and thorax width, forewing length, wingspot length (forewing), total length and abdomen length. All these characters, except wing spot length (see below), were measured with digital callipers (to the nearest $0.01 \mathrm{~mm}$ ), either directly in the field or in the laboratory using individuals that were frozen immediately after capture.

To measure the size of the wing spot, we took photographs of the wings of each male against a common background, using a digital camera (Nikon CoolPix 950). Each photograph also included a reference area of known size $(10 \times 10 \mathrm{~mm})$, which later enabled us to calculate the length, width and area of each individual wing spot (Figure 1). In this paper, we present data only on the length of the forewing spot, because this is the trait that seems to be most important in sexual selection (as revealed by our own field study, see below), and no qualitatively different results would be obtained by instead using the area of the wing spot (E Svensson, L Kristoffersen and Klas Oskarsson, unpublished data).

In addition to the variation in wing spot length, the darkness or saturation of the wing spot colour is important in sexual selection in this and other damselflies (eg Grether, 1996a,b). We therefore estimated the darkness of each male wing spot by classifying males according to a visual scale consisting of seven different classes, with one being almost transparent wings and seven being the darkest, least transparent. This visual scale of wing spot saturation has similarities to the scale used by Grether (1996a,b) in his study on the North American rubyspot damselfly Hetaerina americana. It turned out that most males were classified to classes 2-6 $($ mean $=3.21 \mathrm{SD}=1.28 N=342)$, and much of the variation was age related: immature males had lower colour scores than adult males (2.30 vs 3.96 $\left.F_{1,340}=246.79, P<0.001\right)$. However, even after controlling for age, there was still variation remaining between individual males and populations (see below and 'Results').

To further evaluate the reliability of our visual scale, we compared our wing spot darkness estimates of a set of males consisting of both $C$. splendens $(N=36)$ and its morphologically very similar congener $C$. virgo $(N=34)$, with quantitative measurements of colour using the reflectance spectrometer 'Colourtron'(Hill, 1998). The Colourtron measures three aspects of colour, namely hue $(H)$, brightness $(B)$ and saturation $(S)$ in wavelengths between 390 and $700 \mathrm{~nm}$ (Hill, 1998). By extracting the first principal component (PC1 obtained from a factor analysis) from these three variables, one can then get a reliable composite measure of colour, denoted ' $\mathrm{HSB}^{\prime}$ (Hill, 1998). We compared our visual estimates of male wing spots with this measure of HSB. Classifications of males according to the visual scale were carried out in advance of the use of the Colourtron and were thus independently performed in relation to these latter measurements. Our visual scale was significantly corre- lated with $\operatorname{HSB}\left(F_{1,65}=6.73\right.$; partial $\left.r^{2} 0.10 ; P=0.012\right)$ There was also a significant effect of age on spot darkness $\left(F_{1,65}=23.61\right.$; partial $\left.r^{2} 0.19 ; P<0.001\right)$, but no effect of species $\left(F_{1,65}=0.017\right.$; partial $\left.r^{2} 0.11 ; P=0.90\right)$. These data thus confirm that our visual scale captures a repeatable and important component of wing spot darkness and colour intensity in these damselflies.

\section{Field study of sexual and natural selection}

To directly estimate the strength of current sexual selection in two of our populations, we performed marking-resighting studies in both 2001 (Klingavälsåns Naturreservat) and 2002 (Klingavälsåns Naturreservat and Höje A/Värpinge). Studies were performed between 10 June and 24 July in 2001 (Klingavälsån), between 8 June and 21 August 2002 (Klingavälsån) and between 10 June and 8 August 2002 (Höje Å). We captured and marked as many males as possible during daily visits to the study areas. After capture, morphological measurements were taken from each male, including all the measurements that were recorded in the population survey (see above) and colour classifications of the wing spot darkness were also performed. Phenotypic variances, covariances and correlations between the traits are presented in Supplementary Table 2. We recorded if the male was caught singly or whether he was in copula with a female. Before release, males were marked for future individual identification in the field, by painting the last three abdomen segments in unique three-digit combination, using a waterproof, nonlethal permanent colour (Lefranc and Bourgeois, Le Mans Cedex 2, France).

The two study areas are located $30 \mathrm{~km}$ from each other. Both study plots consist of core areas along the rivers that are approximately $200 \mathrm{~m}$ in length between the end points. At Klingavälsån, there were high densities of both C. splendens and C. virgo, whereas near the upstream and downstream end points, the densities of both these species were much lower (E Svensson, unpublished observations). At Höje $\AA$, there were high densities of C. splendens, but C. virgo did not occur in this area. A major ecological difference between the two populations is thus that $C$. splendens is sympatric with $C$. virgo in Klingavälsån, whereas it is allopatric in Höje $\AA$.

We made regular visits outside the end points at both study areas to search for stragglers that moved outside the core study plots. We also walked through the grasslands surrounding the banks of the river. Very few marked individuals were seen far away from the immediate vicinity of the rivers, and since all matings take place at or close to water, we are confident that our field observations provide good estimates of both natural and sexual selection in these populations. We only used males that were resighted in the field at least once after they had been marked and released, to avoid biases due to differential dispersal arising from postmarking trauma.

Each day, 1-3 persons walked repeatedly and regularly both upstream and downstream the river in search of marked individuals. Field work was carried out every day during the study period between 1000 and 1700 hours, which is the main mating period of these damselflies. No field work was carried out in days with heavy rain and/or strong wind, since Calopteryx are very 
sensitive to these weather conditions and remain totally inactive under such circumstances. During our visits to the study plot, we recorded all resighted marked males and we also noted if males were observed alone, if they were copulating or if they were visited by one or several females.

Admittedly, we must have missed a number of copulations since we could not observe all the males during their whole life spans. However, this will not cause any bias in our sexual selection estimates as long as the copulations we missed were not biased with respect to variation in male phenotypic characters. This is because selection differentials and selection gradients are defined as the covariance between relative fitness and phenotypic traits, rather than in terms of absolute magnitude of fitness (Lande and Arnold, 1983). To estimate variation among individual males in their mating rate (no. copulations/hour), we performed detailed focal behavioural observations on each of the resighted males $(N=153,184$ and 176 for Klingavälsån 2001, 2002 and Höje $\AA$ 2002, respectively). The length of the focal observation periods ranged from 1 to $201 \mathrm{~min}$ (means \pm SEs: Klingavälsån $=18.77 \pm 1.38 ;$ Höje $\AA=$ $26.69 \pm 1.91)$. For detailed discussion about methodology in recording male mating success, see Grether (1996a,b) and Koenig and Albano (1987).

\section{Molecular analyses}

Collected specimens $(N=179)$ were decapitated and heads were placed in $200 \mu \mathrm{l}$ lysis buffer $(0.1 \mathrm{M}$ Tris, 0.005 EDTA, $0.2 \%$ SDS, $0.2 \mathrm{M} \mathrm{NaCl}$, pH 8.5) with $6 \mu \mathrm{l}$ proteinase $\mathrm{K}(10 \mathrm{mg} / \mathrm{ml})$ for $3 \mathrm{~h}$ of digestion at $55^{\circ} \mathrm{C}$ (Laird et al, 1991), followed by standard ethanol precipitation. The precipitates were suspended in $500 \mu \mathrm{l}$ $\mathrm{ddH}_{2} \mathrm{O}$ and the DNA was isolated following a standard phenol/chloroform extraction protocol (Laird et al, 1991). The DNA concentration was checked on a spectrophotometer and the samples were diluted to a concentration of approximately $50 \mathrm{ng} / \mu \mathrm{l}$.

We used the AFLP protocol of Vos et al (1995) with only minor modifications as described in Bensch et al (2002). The fragments were separated in $6 \%$ polyacrylamide gels and detected by fluorescein-labelled E-primers in a Vistra FluorImager. We used three selective primer combinations: $\mathrm{A}\left(\mathrm{E}_{\mathrm{TAG}} \times \mathrm{M}_{\mathrm{CAC}}\right), \mathrm{B}\left(\mathrm{E}_{\mathrm{TGA}} \times \mathrm{M}_{\mathrm{CCG}}\right)$ and $\mathrm{C}\left(\mathrm{E}_{\mathrm{TAG}} \times \mathrm{M}_{\mathrm{CAC}}\right)$. These primer combinations amplified relatively few but easily scorable bands (Figure 2 ), and we restricted the evaluation to variable bands that could be scored unequivocally: 24 for primer combination A, 11 for primer combination $\mathrm{B}$ and 27 for primer combination C. AFLP analysis thus enables workers to rapidly assess molecular variation at a large number of nuclear loci (62 in the present study). The vast majority (>95\%) of these nuclear loci will be noncoding and hence be selectively neutral. The large number of loci that can be assessed using this protocol partly compensates for the fact that it is only possible to distinguish the presence or absence of one allele per locus. Hence, AFLP is a dominant molecular marker and those with a band can be either heterozygotes or homozygotes for the presence-allele.

\section{Statistical analyses}

Data on molecular variation were analysed using Arlequin ver. 2.0 (Excoffier et al, 1992). We calculated

\section{Individual \#}

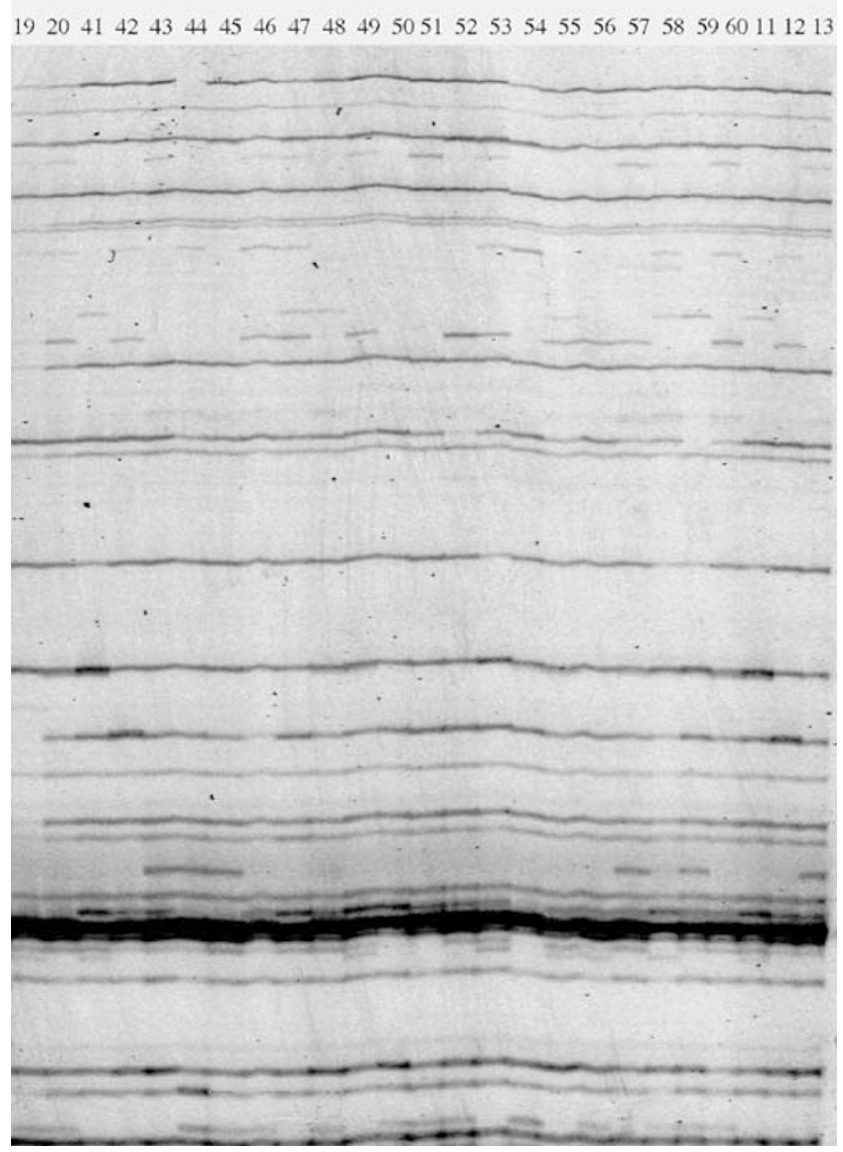

Figure 2 Amplified fragment length polymorphism profiles in $25 \mathrm{C}$. splendens using primer combination $\mathrm{A}$. The fragments were separated in polyacrylamid gels and visualized by reading the fluorescein-labelled E-primers in a Vistra FluorImager.

an overall $F_{\text {st }}$-value among all the populations, and partitioned this $F_{\mathrm{st}}$-value to variation among rivers and among populations within rivers, using nested molecular analysis of variance (AMOVA). We obtained a standard error around the mean for the overall $F_{\mathrm{st}}$-value, by separately estimating locus-specific $F_{\text {st }}$-values for all of the 62 loci investigated, and subsequently obtaining bootstrapped estimates of confidence limits (CLs), using a standard programme written in Resampling Stats (Simon, 2000).

All data on morphological measurements from our population survey were analysed using STATISTICA (Statsoft, 2000). We performed multivariate analysis of variance (MANOVA) to investigate if population centroids of trait means differed significantly from each other. These multivariate analyses were subsequently followed up by simple univariate analysis of variance (ANOVA) of the individual, separate traits. To obtain quantitative estimates of population divergence for the morphological traits, we used nested (hierarchical) ANOVAs to extract both absolute and relative (\%) variance components and partition variation between rivers, between populations within rivers and within populations. These analyses were performed using VARCOMP module in STATISTICA, and we also 
visualized the absolute and relative variance components for the traits. We used the summed relative variance components explained by rivers and populations as a quantitative measure of morphological population divergence (the remaining variance was explained by variation among individuals within populations). The absolute variance component analysis was based on squared units of the original variables ( $\mathrm{mm}$ for all traits, except wing spot darkness), whereas the relative variance component analysis was dimensionless (ie percentage-wise variation).

We compared molecular and morphological population divergence by calculating the correlation between pairs of populations for molecular (Pairwise $F_{\text {st }}$-values between populations) and phenotypic data (Mahalanobis distances between populations calculated by taking all the phenotypic traits into account). We studied 12 populations and there were 66 such pairwise population comparisons. Since each population was involved in several of these comparisons, the conventional probability values for the correlation coefficients are not reliable due to statistical nonindependence. We therefore estimated $P$-values using Mantel tests, a resampling procedure that is especially appropriate for spatial data sets of this kind (Raymond and Rousset, 1995). Similarly, we used Mantel tests to estimate $P$-values for the correlation coefficients of isolation-by-distance (pairwise distances between pairs of populations) for both molecular and the phenotypic data.

To quantify the strength of sexual selection from our field data of marked individuals, we calculated univariate standardized selection differentials using parametric regression analysis (Lande and Arnold, 1983; Endler, 1986). Prior to analysis, all traits were standardized to mean zero and unit variance. In this study, we used two fitness estimates: male mating rate (representing sexual selection) and lifespan (representing natural selection). Both our fitness estimates were divided by mean male fitness to obtain a measure of relative fitness. Selection differentials were then calculated as the covariance between relative fitness and standardized trait values.

Selection differentials provide information about the total strength of selection on a character, and thus include both direct selection and indirect selection arising through covariances with other characters. A problem with the interpretation of univariate selection differentials is that selection may not act directly on the focal trait but instead on correlated characters (Lande and Arnold, 1983). In particular, natural or sexual selection may favour larger overall body size or 'condition' (Houle, 1991). This may then lead to a confounding positive covariance between fitness and traits that are merely correlated with body size (or condition), and as a consequence selection on such correlated traits will be overestimated (see Lande and Arnold, 1983; Houle, 1991). To separate the effects of direct selection on our traits from the effects of indirect selection on body size and other traits, we followed up our univariate analyses by performing multiple regressions in which we included all the traits that had selection differentials that were significant or close to significant (ie $P<0.10$ ), as well as a measure of overall body size (PC1; see further below). A measure of structural body size was obtained from the first principal component (PC1) from a factor analysis of the five size-related traits (wing length, total length, abdomen length, thorax length and thorax width).

Although our sexual selection estimate was nonnormally distributed due to high mating skew among males, this will not affect the parametric coefficients in selection analyses (Lande and Arnold, 1983). However, $P$-values may become affected if errors are non-normally distributed in parametric regression analysis, and it has therefore been recommended to confirm probability values using alternative statistical methods such as generalized linear models (Mitchell-Olds and Shaw, 1987). We followed these recommendations and obtained $P$-values for the selection coefficients by performing generalized linear models, assuming a poisson error distribution and using a probit link function (VGLZ module in STATISTICA). Probability values reported are those from likelihood type 3-tests (Statsoft, 2000).

\section{Results}

\section{Population divergence in morphology}

A MANOVA with all the traits as dependent variables, revealed that the 12 populations differed significantly in their overall morphology, as revealed by differences in population centroids (Wilk's $\lambda_{77,1883.1}=0.311 P<0.001$ ). Further univariate analyses of the different traits revealed that the trait means were highly significant between the different populations (ANOVAs $P<0.001$ for all traits). Population means for the morphological traits are shown in Figure 3. To investigate if trait differences among populations were confounded by overall size differences, we repeated the analysis using a MANOVA with population as a factor and PC1 ('size') as a covariate. All the investigated traits differed significantly in this new analysis as well $\left(P^{\prime} S<0.001\right)$, indicating that overall body size differences among the populations did not confound our previous results.

We calculated differences (in SD units) in morphology between populations with the lowest and those with the highest trait values. The difference between the population with the lowest and the highest trait mean was typically between 1 and 2 SD units (Table 1). Total body length was the trait that was most highly differentiated among populations (1.97 SDs), whereas spot darkness was the trait that was least differentiated (1.24 SDs, Table 1; Figure 4). Note, however, that somewhat different results are obtained for absolute population divergence (Figure 4) as compared to the standardized difference in relative population divergence (Figure 5). The relative population divergence takes into account not only the differences in trait means between populations but also the amount of within-population variation, which was quite high for spot darkness.

\section{Selection study}

We were able to follow a total of 513 individually marked males during 2001 and 2002. These males were observed at least once in the field after they had been captured and marked. The mean lifespan of these males (days between capture and last observation) was 4.78 $( \pm 0.21)$ days. Of these 513 males, 40 copulated at least once (mean copulation rate/hour $( \pm S E$ ) among these males $=2.48 \pm 0.19)$. 


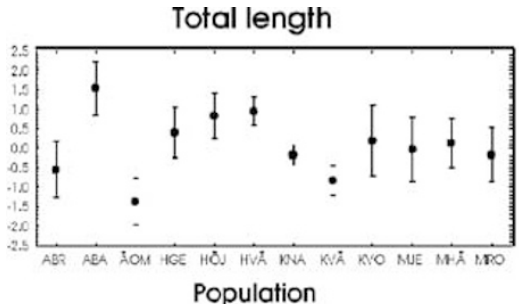

Abdomen length

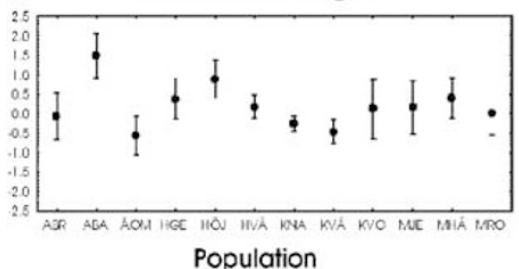

Thorax length

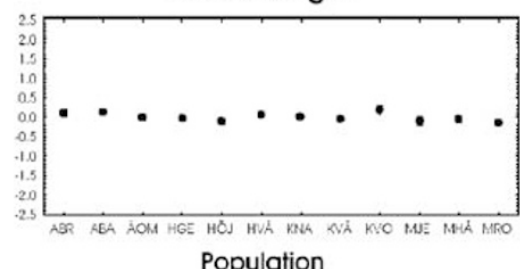

Forewing length

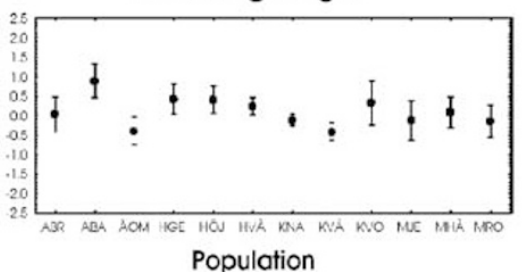

Population

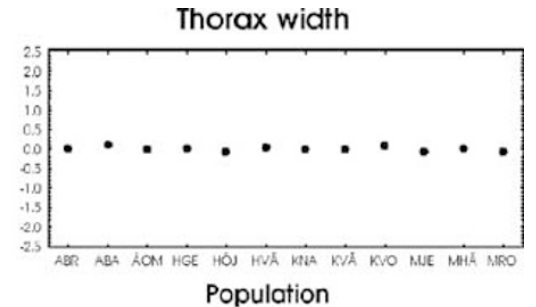

Population

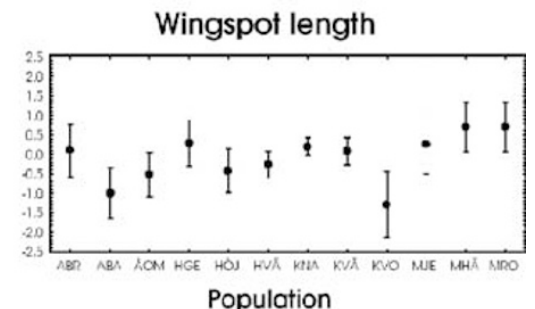

Wingspot darkness

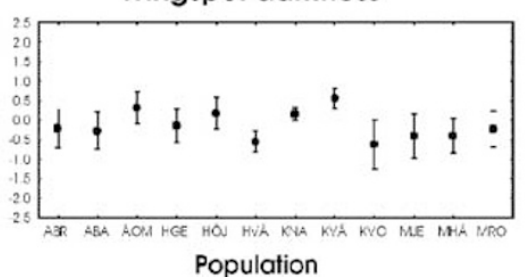

Figure 3 Population divergence in morphology: trait means $( \pm$ SEs). Differences between populations in trait means are highly significant (see Results). Effects of age (mature vs immature) were removed prior to analysis (age-corrected residuals are shown). Abbreviations for different populations are provided. For full names of populations and localities, see Supplementary Tables 1 and 2.

Table 1 Absolute $(Z)$ and relative ( $\Delta Z$ : standard deviation units) population divergence for eight morphological traits among 12 Calopteryx populations in southern Sweden

\begin{tabular}{|c|c|c|c|c|c|c|c|c|}
\hline \multirow[t]{2}{*}{ Trait } & \multirow[t]{2}{*}{$\mathrm{Z}$} & \multirow[t]{2}{*}{$\Delta \mathrm{Z}$} & \multicolumn{3}{|c|}{ Sexual selection: mating rate } & \multicolumn{3}{|c|}{ Natural selection: survival } \\
\hline & & & $\mathrm{S}_{\text {Klinga2001 }}$ & $\mathrm{S}_{\text {Klinga2002 }}$ & $\mathrm{S}_{\text {Höje2002 }}$ & $\mathrm{S}_{\text {Klinga2001 }}$ & $\mathrm{S}_{\text {Klinga2002 }}$ & $\mathrm{S}_{\text {Höje2002 }}$ \\
\hline Spot darkness & 1.72 & 1.24 & 0.116 & 0.055 & -0.203 & 0.034 & -0.006 & -0.130 \\
\hline Forewing spot length & 2.25 & 1.54 & $0.361^{\dagger}$ & $-0.334^{*}$ & $0.350^{*}$ & -0.040 & -0.018 & 0.011 \\
\hline Forewing length & 1.20 & 1.51 & 0.199 & -0.016 & 0.022 & $-0.155^{*}$ & 0.076 & -0.058 \\
\hline Total length & 2.93 & 1.97 & -0.297 & $-0.354^{*}$ & 0.170 & $-0.198^{* *}$ & $-0.159 *$ & -0.060 \\
\hline Abdomen length & 2.20 & 1.74 & -0.222 & $-0.461^{* *}$ & 0.027 & -0.028 & 0.099 & -0.061 \\
\hline Thorax length & 0.32 & 1.88 & -0.038 & -0.209 & -0.101 & 0.082 & $-0.183^{*}$ & -0.071 \\
\hline Thorax width & 0.19 & 1.54 & 0.097 & 0.055 & $0.416^{*}$ & 0.095 & $-0.192^{* *}$ & 0.022 \\
\hline PC1 ('Size') & 1.27 & 1.27 & -0.250 & -0.106 & 0.109 & 0.090 & -0.173 & -0.075 \\
\hline
\end{tabular}

We also present sexual and natural selection differentials obtained in a field study on two of these populations. All selection gradient with $P$-values $<0.10$ are indicated in bold. Symbols: ${ }^{* * *}=P<0.001 ;{ }^{* *}=P<0.01 ;{ }^{*}=P<0.05 ;{ }^{\dagger}=P<0.10$.

Estimates of natural and sexual selection differentials for the seven traits as well as for PC1 are presented in Table 1. They range in absolute magnitude from 0.006 (natural selection on spot darkness at Klingavälsån, 2002) to 0.461 (sexual selection on abdomen length at Klingvälsån, 2002). The statistical power in selection analyses is known to be very low (Mitchell-Olds and Shaw, 1987), which has probably caused a bias in the published literature on selection coefficients (Endler, 1986; Hoekstra et al, 2001). For completeness, we therefore present the selection differentials for all of the traits in this study. Note especially that selection differentials were significant or close to significant for forepatch length in both populations, and that selection on this trait changed sign between 2001 and 2002 at Klingavälsån (see also Table 2). Interestingly, sexual selection was found to be stronger than natural selection, when comparing the two types of selection differentials for the two populations and years (average sexual selection differential $=0.190$; average natural selection differential $=0.088$; Paired $t$-test: $t=3.384$, d.f. $=23, P=0.0026)$. This might suggest that sexual selection could be a more powerful force than natural selection in population divergence. Alternatively, the fact that sexual selection is measured over shorter time intervals (minutes and hours) than natural selection (days) can explain such a difference, a pattern that has also been documented in a recent large meta-analysis of published selection coefficients (Hoekstra et al, 2001). 
Finally, in Table 3, we summarize the trait differences and net selection gradients between the two study populations. Although these populations are located close to each other, and only weakly differentiated at the AFLP-loci (pairwise $F_{\text {st }}=0.001$; Table 4 ), they are highly differentiated in seven of the eight morphological traits (Table 3).

Results from our multiple regressions and estimates of multivariate selection gradients $\left(\beta^{\prime}\right.$ s) are presented in Table 2. The general picture that emerges is that our univariate selection differentials were not confounded, either by selection on body size or selection on correlated characters.

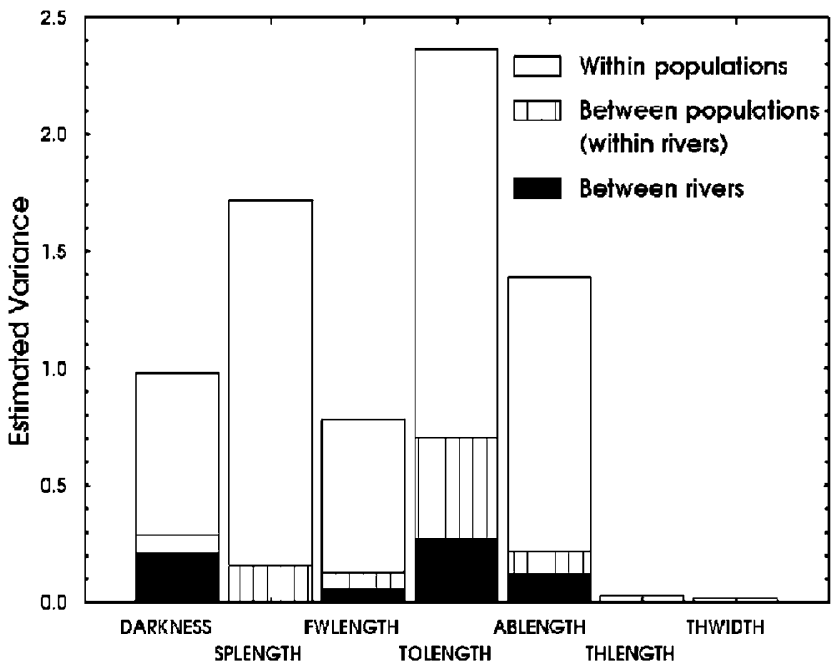

Figure 4 Population divergence in morphology: absolute variance components. Variance components obtained from a nested ANOVA (see Results). Trait abbreviations: SPOTDARK (wingspot darkness), SPLENGTH (wingspot length), WILENGTH (forewing length), TOLENGTH (total length), ABLENGTH (abdomen length), THLENGTH (thorax length), THWIDTH (thorax width).
Molecular population differentiation

Nested AMOVA revealed significant among-population differentiation in the 62 AFLP loci $\left(F_{\text {st }}=0.054\right.$. $\left.P<0.001\right)$. Total molecular variation could be partitioned to variation between rivers $(4.27 \%)$, between populations within rivers $(1.16 \%)$ and between individuals within populations $(94.57 \%)$. Pairwise $F_{\mathrm{st}}$-values between populations ranged from $\sim 0$ to 0.13 , and several of these population differences were significant (Table 4 ).

Within populations

Between rivers

Between populations (within rivers)

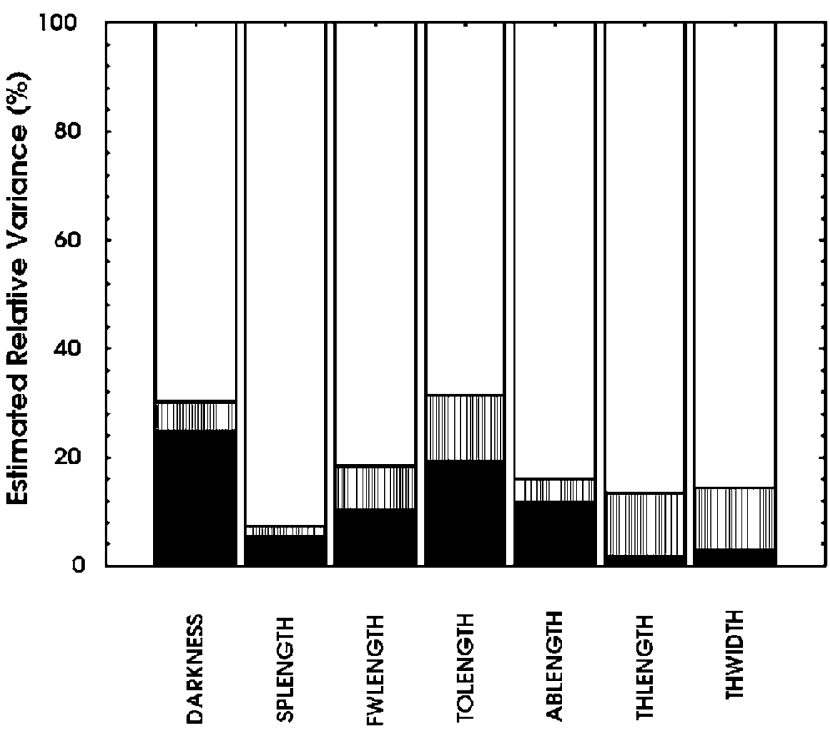

Figure 5 Population divergence in morphology: relative variance components (\%). Variance components obtained from a nested ANOVA (see Results). For explanations of trait abbreviations, see Figure 4.

Table 2 Standardized selection gradients ( $\beta$ :as), SEs and $P$-values from multiple regression analyses in which traits with significant selection differentials (see Table 1) were incorporated alongside with PCI, a measure of structural body size

\begin{tabular}{|c|c|c|c|c|c|}
\hline Type of selection & Population (year) & Trait & $\beta$ & $S E$ & $\mathrm{P}$ \\
\hline \multirow[t]{9}{*}{ Sexual: mating rate } & \multirow[t]{2}{*}{ Klingavälsån (2001) } & Forewing spot length & $0.422^{*}$ & 0.201 & 0.035 \\
\hline & & PC1 ('size') & -0.240 & 0.220 & 0.28 \\
\hline & \multirow[t]{4}{*}{ Klingavälsån (2002) } & Forewing spot length & $-0.328^{*}$ & 0.162 & 0.043 \\
\hline & & Total length & -0.404 & 0.310 & 0.19 \\
\hline & & Abdomen length & $-0.678^{* *}$ & 0.239 & 0.004 \\
\hline & & PC1 ('size') & $0.689^{*}$ & 0.321 & 0.032 \\
\hline & \multirow{3}{*}{ Höje $\AA$ (2002) } & Forewing spot length & $0.324^{\dagger}$ & 0.176 & 0.065 \\
\hline & & Thorax width & $0.568^{*}$ & 0.232 & 0.014 \\
\hline & & PC1 ('size') & 0.310 & 0.219 & 0.16 \\
\hline \multirow{9}{*}{ Natural: Life-span } & \multirow[t]{3}{*}{ Klingavälsån (2001) } & Forewing length & $-0.230^{*}$ & 0.114 & 0.044 \\
\hline & & Total length & $-0.331^{*}$ & 0.131 & 0.012 \\
\hline & & PC1 ('size') & $-0.324^{*}$ & 0.153 & 0.034 \\
\hline & \multirow[t]{4}{*}{ Klingavälsån (2002) } & Total length & -0.216 & 0.185 & 0.24 \\
\hline & & Thorax length & -0.212 & 0.143 & 0.14 \\
\hline & & Thorax width & $-0.218^{\dagger}$ & 0.130 & 0.096 \\
\hline & & PC1 ('size') & 0.367 & 0.303 & 0.228 \\
\hline & \multirow[t]{2}{*}{ Нӧје $\AA$ (2002) } & Spot darkness & $\mathbf{0 . 1 3 3}$ & 0.072 & 0.064 \\
\hline & & PC1 ('size') & -0.081 & 0.070 & 0.25 \\
\hline
\end{tabular}

Selection gradients estimate direct selection on these traits when controlling for selection on the other traits that are incorporated in the analysis (eg body size). Hence, significant selection gradients in this analysis are not confounded by selection on body size. All selection gradient with $P$-values $<0.10$ are indicated in bold. Symbols: ${ }^{* * *}=P<0.001 ;{ }^{* *}=P<0.01{ }^{*}=P<0.05{ }^{*}{ }^{\dagger}=P<0.10$. 
Morphology, molecular markers and isolation-by-distance Nested (hierarchical) ANOVAs were used to extract variance components and partition morphological variance to variation between rivers, between populations within rivers and within populations in a similar way as we partitioned molecular variance above. These analyses revealed that the two traits that were most variable (in absolute terms) were total length and forespot wing length, whereas thorax length and thorax width were the least variable traits (Figure 4). Although most of the variation was between individuals within populations, $10-30 \%$ of the variation was due to variation between rivers and between populations within rivers (Figure 5).

There was a significant correlation between phenotypic and molecular population divergence $(r=0.39$; $P=0.005$; Figure 6a). Thus, differences between pairs of populations for the AFLP data was mirrored in a similar difference in their phenotypic means (Figure 6a). In addition, we found evidence for significant isolation-bydistance for the molecular data $(r=0.70 ; P<0.001$; Figure $6 \mathrm{~b})$, but not for the phenotypic traits $(r=0.42$; $P=0.33$; Figure 6c).

\section{Discussion}

Population divergence in morphology in these populations of $C$. splendens is likely to be adaptive since the

Table 3 Pairwise population comparison (Höje Å vs Klingavälsån) of sexual selection and morphology

\begin{tabular}{|c|c|c|c|}
\hline Trait & $\begin{array}{l}\text { Net selection } \\
\text { differential }\end{array}$ & $\begin{array}{l}\text { Trait difference } \\
\quad(\text { absolute })\end{array}$ & $\begin{array}{l}\text { Trait difference } \\
\text { (SD units) }\end{array}$ \\
\hline Spot darkness & 0.118 & $-1.688^{* *}$ & $-1.120^{* *}$ \\
\hline Forewing spot length & 0.336 & $0.632^{* * *}$ & $0.456^{* * *}$ \\
\hline Forewing length & -0.070 & 0.037 (NS) & 0.041 (NS) \\
\hline Total length & 0.496 & $0.766^{* * *}$ & $0.598^{* * *}$ \\
\hline Abdomen length & 0.368 & $0.464^{* * *}$ & $0.484^{* * *}$ \\
\hline Thorax length & 0.022 & $0.049 * *$ & $0.250^{* *}$ \\
\hline Thorax width & 0.340 & $-0.167^{* *}$ & $-0.933^{* *}$ \\
\hline PC1 ('size') & 0.287 & $0.247^{* *}$ & $0.247^{* *}$ \\
\hline
\end{tabular}

In this table, net selection differentials (ie selection differential values for Höje $\AA$ minus those for Klingavälsån) for eight morphological traits are shown. Raw data are presented in Table 1. Significance tests for trait differences between populations were obtained from an MANOVA. Symbols: ${ }^{* * *}=P<0.001 ;{ }^{* *}=P<0.01$ ${ }^{*}=P<0.05 ; \quad+=P<0.10$. Significant differences in trait means $(P<0.05)$ are indicated in bold. amount of observed divergence could quickly be generated by the strength of current selection that we have estimated in two of our populations (Tables 1-3). We do not have heritability estimates for any of the phenotypic traits that we have studied, but our finding of a significant correlation between molecular and phenotypic population divergence (Figure 6a) indicates that the between-population component of trait variation is at least partly heritable.

How could such a correlation between morphological and molecular population divergence be generated? The most likely explanation for this pattern is that AFLP loci we have studied $(N=62)$ are either loci coding for the morphological traits or, perhaps more likely, that they are linked to other loci coding for the morphological traits. If so, the AFLP loci investigated in this study will not strictly behave as neutral markers since they will then be subject to indirect selection due to their association with loci under direct selection (ie those affecting morphology). Alternatively, stabilizing selection on morphology around a common mean and different degree of gene flow between pairs of populations could generate such a correlation between morphological and molecular distance, hence population divergence in both measures may partly reflect the current degree of gene flow. Our data are consistent with an important role for selection in population differentiation, although we cannot exclusively determine whether natural or sexual selection has been most important. However, since sexual selection on the traits we have investigated seems to be stronger than natural selection (Table 1; see Results for statistics), we believe that its role in population differentiation is likely to be very important.

Studies quantifying the discordance or concordance between morphological and molecular population divergence are certainly informative (Merilä, 1997; Merilä and Crnokrak, 2001; Figure 6a). They do, however, have several limitations. First, it is impossible to distinguish between different kinds of selection (natural vs sexual) in such morphology-molecular comparisons (Schluter, 2000). Another important concern is the uncertainty of the genetic basis of the phenotypic characters (Lewontin, 1984; Rogers, 1986; Schluter, 2000). The morphological traits we have studied are likely to be under polygenic control and governed by multiple loci. Indirect inferences about the action of selection based on discrepancies between molecular and morphological divergence can never reveal the selective agents or ecological causes

Table 4 Pairwise $F_{\text {st }}$ values between the 12 study populations

\begin{tabular}{|c|c|c|c|c|c|c|c|c|c|c|c|}
\hline Population & $A B R$ & $A B A$ & AOM & $H G E$ & HÖJ & $H V \ddot{A}$ & $K N A$ & $K V \ddot{A}$ & $K V O$ & MJE & $M H \ddot{A}$ \\
\hline ABA & 0.001 & & & & & & & & & & \\
\hline ÅOM & 0.062 & 0.085 & & & & & & & & & \\
\hline HGE & 0.030 & 0.068 & 0.090 & & & & & & & & \\
\hline HÖJ & 0.070 & 0.051 & 0.096 & 0.047 & & & & & & & \\
\hline HVÄ & 0.033 & 0.027 & 0.019 & 0.097 & 0.106 & & & & & & \\
\hline KNA & 0.009 & 0.032 & 0.015 & 0.075 & 0.085 & 0.001 & & & & & \\
\hline KVÄ & 0.035 & 0.033 & 0.011 & 0.096 & 0.105 & 0.021 & 0.000 & & & & \\
\hline KVO & 0.000 & 0.014 & 0.026 & 0.080 & 0.085 & 0.021 & 0.021 & 0.000 & & & \\
\hline MJE & 0.018 & 0.051 & 0.040 & 0.010 & 0.007 & 0.070 & 0.041 & 0.051 & 0.041 & & \\
\hline MHÄ & 0.059 & 0.079 & 0.109 & 0.027 & 0.061 & 0.128 & 0.084 & 0.093 & 0.087 & 0.022 & \\
\hline MRO & 0.030 & 0.078 & 0.047 & 0.053 & 0.073 & 0.085 & 0.058 & 0.063 & 0.047 & 0.023 & 0.010 \\
\hline
\end{tabular}

For explanation of populations abbreviations, see Supplementary Tables 1 and 2 . Significant $(P<0.05)$ pairwise $F_{\mathrm{st}} \mathrm{s}$ are indicated in bold (significance tests obtained from permutation tests). 


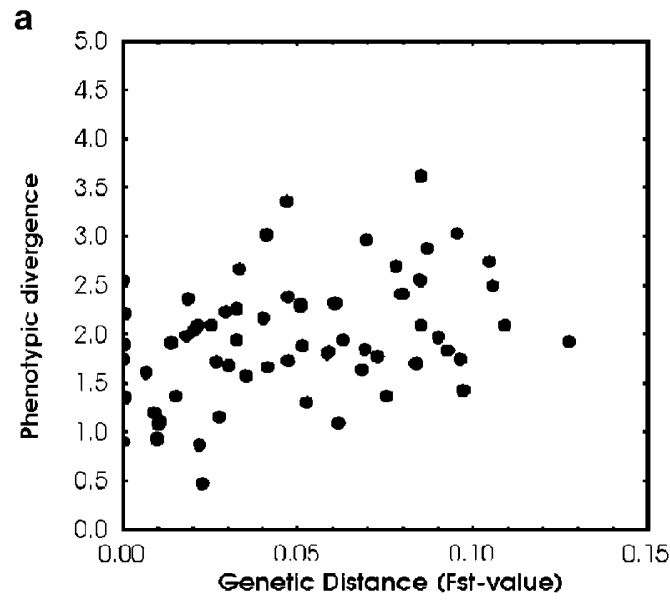

b
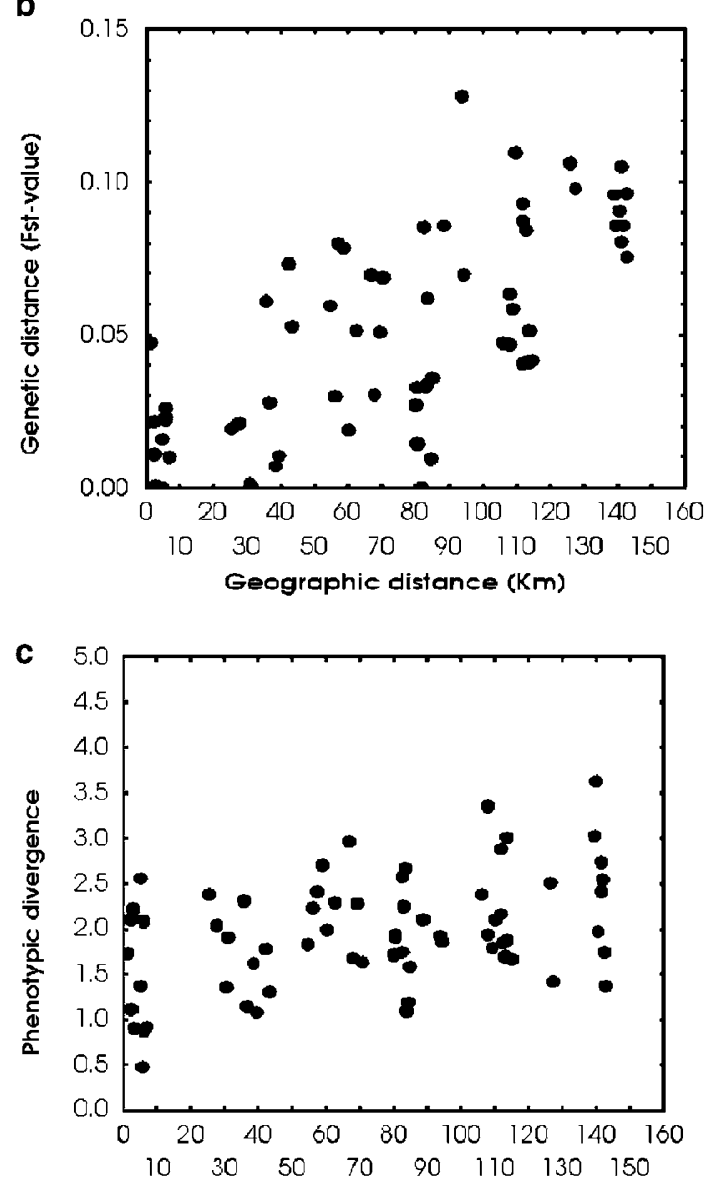

Geographic Distance (Km)

Figure 6 Comparisons between morphological and molecular population divergence and isolation-by-distance. Data points are pairwise comparisons between sets of 12 populations, and differences are tested with Mantel tests (see text for details). (a) Morphological distances between populations are significantly correlated with molecular distances estimated as pairwise

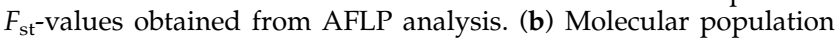
divergence shows significant evidence of isolation-by-distance. (c) There is no significant evidence of isolation-by-distance for morpology.

of selection, and should therefore only be complementary to approaches in which selection is measured directly in the wild (Lande and Arnold, 1983; Wade and Kalisz, 1990; Schluter, 2000). Studies in which direct measurements of selection in multiple populations are combined with estimates of molecular population divergence, like in the current study, are clearly needed (Merilä and Crnokrak, 2001).

The strong correlation $(r=0.70)$ between genetic and geographic distances between populations (Figure 6b) provides empirical support to the classical isolation-bydistance model originally developed by Wright (1943). Such a pattern of genetic differentiation of populations can arise if dispersal and gene flow occur primarily between nearby and adjacent populations, such as between sites within a river drainage, and more rarely between different river systems. A similar pattern of isolation-by-distance, albeit weaker correlation $(r=0.295)$, has also been found among populations of the stonefly Peltoperla tarteri (Schultheis et al, 2002). Furthermore, our finding of greater molecular variation among than within rivers, as revealed by the nested AMOVA, is similar to the pattern found in another species of aquatic stonefly (Yoraperla brevis; Hughes et al, 1999).

Several of our sexual selection estimates in this study are very high, compared to the sexual selection coefficients in a recent meta-analysis (Hoekstra et al, 2001). The sexual selection differentials for wingspot length varied between -0.334 and 0.350 , which is slightly lower than the estimates reported by Grether in his sexual selection study of the North American rubyspot damselfly (Grether, 1996a,b). Although we cannot disentangle the role of intra- vs intersexual selection, our data indicate an important role for sexual selection as a major driving force in population differentiation of C. splendens. Strong selection in local populations could potentially lead to peak shifts and partial sexual isolation via correlated responses in mate preferences (Tilley et al, 1990; Rice and Hostert, 1993; Price et al, 1993). Furthermore, if selection fluctuates between generations, populations may get out of phase with each other and rapidly become reproductively isolated (Iwasa and Pomiankowski, 1995). In this context, it is interesting that sexual selection changed in both sign and magnitude between 2001 and 2002 at Klingavälsån (Tables 1 and 2). Finally, strong sexual selection will also reduce the local effective population sizes and hence increase the potential for genetic drift and population differentiation at neutral or nearly neutral loci (Table 4).

Although we detected pronounced population divergence in the AFLP loci in this study (Table 4), about 95\% of the molecular genetic variation was attributable to variation among individuals within populations. Gene flow could easily counteract genetic drift in local populations and limit neutral population divergence, but we doubt that it would have a strong influence in limiting the substantial population divergence we have detected for the adaptive characters (Figures 2-5), particularly in light of the strong sexual selection (Tables $1-3)$. It seems more likely that sexual selection fluctuates between generations and differs between populations and it may also sometimes be counteracted by survival selection. For instance, males with small patches may be favoured in certain years, or females may not prefer large-patched males in all populations. The variability in the strength and magnitude of sexual selection, both between populations and years (Tables 1-3) support such an interpretation. The net result of this would then be long-term conflicting selection pressures on the traits and 
different locations of the population-specific optima. Lande (1982) showed that the inherent genetic instability of mating systems in local populations could quickly lead to rapid male character divergence, sexual isolation and speciation over a continuous geographic range. He assumed, however, that there was no direct selection acting on female preferences in this model (Lande, 1982). Subsequent theoretical work have revealed that such local runaway processes could indeed work even if mate preferences are subject to direct selection, if spatial effects are considered and females have open-ended preferences (Payne and Krakauer, 1997; Day, 2000; Hall et al, 2000).

We found that body length, abdomen length, wingspot darkness, wingspot length and wing length were the traits that had diverged most, and thorax length and thorax width had diverged least (Figure 3). The limited amount of absolute population divergence in the thorax traits (Figure 4) may be explainable in terms of strong canalizing selection on these traits. This fits well into the classical view that sexually selected traits are diverging faster than naturally selected traits (Lande, 1981; WestEberhard, 1983; Rice and Holland, 1997). As a caveat, we note that the relative variance portioning analysis suggested that the thorax traits diverge in a similar way to the other traits (Figure 5), which raises the question of which of the two variance partitioning approaches that is most useful in quantifying population divergence. Wing colouration, body size and wing length have been shown to be important traits in both intrasexual selection (male combats for territories) and intersexual selection (female choice) (Plaistow and SivaJothy, 1996; Grether, 1996a, b; Siva-Jothy, 1999; Plaistow and Tsubaki, 2000). In contrast, thorax length and width are likely to be subject primarily to natural selection, for example, for improved flight capacity to avoid avian predation. The wing patch characters, patch length and patch darkness, in Calopteryx are particularly interesting because of the potential for conflicting selection pressures between intraspecific sexual selection (Siva-Jothy, 1999; this study), their role in species recognition (Waage, 1979) and the role of natural selection from avian predators on patch characteristics. Avian predation on Calopteryx from pied wagtails (Motacilla alba) is higher at Klingavälsån than Höje A, judged by a higher number of daily observations of these birds and extensive occurrence of wing remnants at 'slaughter places' at the former locality (E Svensson, unpublished data). The consequences of these population differences will be presented in detail in a future paper.

Theory suggests that speciation is facilitated if the signalling traits involved in incipient species recognition are themselves subject to direct natural and/or sexual selection and hence do not have to rely only on indirect selection through their association with other fitnessrelated characters (Felsenstein, 1981; Rice and Hostert, 1993). This situation may apply to the wing spots of several Calopteryx-species both in North America and Europe, and one of the classical cases of reproductive character displacement is the one between females of C. aequabilis and C. maculata (Waage, 1979).

\section{Acknowledgements}

We are grateful to Bengt Hansson, Darren Irwin and Lars Råberg for constructive comments and criticisms on an early draft of this manuscript. We also thank the many field assistants that have helped us with daily observations during the summers 2001 and 2002. This study was financially supported by grants from the Swedish Research Council (VR; to E Svensson and S Bensch) and from The Royal Swedish Academy of Sciences, Stiftelserna Oscar och Lilli Lamms Minne and Lunds Djurskyddsfond (to E Svensson).

\section{References}

Andersson M (1994). Sexual Selection, Princeton University Press: Princeton, NJ.

Anholt BR (1991). Measuring selection on a population of damselflies with a manipulated phenotype. Evolution 45: 1091-1106.

Askew RR (1988). The Dragonflies of Europe, B.H. and A. Harley Ltd: Colchester, UK.

Bensch S, Heidrich P, Salomon M, Seibold I (2002). AFLP analysis identifies hybrids between two subspecies of warblers. Mol Ecol 11: 473-481.

Bohonak AJ, Jenkins DG (2003). Ecological and evolutionary significance of dispersal by freshwater invertebrates. Ecol Lett 6: 783-796.

Butlin R (1987). Speciation by reinforcement. Trends Ecol Evol 2: 8-13.

Butlin R (1989). Reinforcement of pre-mating isolation. In: Otte D, Endler JA (eds) Speciation and its Consequences, Sinauer: Sunderland. pp 158-179.

Charlesworth B, Lande R, Slatkin M (1982). A neo-Darwinian commentary on macroevolution. Evolution 36: 474-498.

Corbet PS (1999). Dragonflies: Behaviour and Ecology of Odonata, Harley Books: Colchester, UK.

Coyne JA, Barton NB, Turelli M (1997). Perspective: a critique of Sewall Wright's shifting balance theory of evolution. Evolution 51: 643-671.

Dale S, Slagsvold T, Lampe HM, Saethre GP (1999). Population divergence in sexual ornaments: the white forehead patch of Norwegian pied flycatchers is small and unsexy. Evolution 53: 1235-1246.

Darwin C (1871). The Descent of Man and Selection in Relation to Sex 1 edn. Murray: London, UK.

Day T (2000). Sexual selection and the evolution of costly female preferences: spatial effects. Evolution 54: 715-730.

Endler JA (1986). Natural Selection in the Wild, Princeton University Press: Princeton, NJ.

Excoffier L, Smouse PE, Quattro JM (1992). Analysis of molecular variance inferred from metric distances among DNA haplotypes: application to human mitochondrial DNA restriction data. Genetics 131: 479-491.

Fear KK, Price T (1998). The adaptive surface in ecology. Oikos 82: $440-448$.

Felsenstein J (1981). Skepticism towards Santa Rosalia, or why are there so few kinds of animals? Evolution 35: 124-138.

Fincke OM (1986). Lifetime reproductive success and the opportunity for selection in a nonterritorial damselfly (Odonata: Coenagrionidae). Evolution 40: 791-803.

Fisher RA (1930). The Genetical Theory of Natural Selection, Oxford: Clarendon Press.

Geenen S, Jordaens K, De Block M, Stoks R, De Bruyn L (2000). Genetic differentiation and dispersal among populations of the damselfly Lestes viridis (Odonata). J North Am Benthol Soc 19: 321-328.

Grether GF (1996a). Intrasexual competition alone favors a sexually dimorphic ornament in the rubyspot damselfly Hetaerina americana. Evolution 50: 1949-1957.

Grether GF (1996b). Sexual selection and survival selection on wing colouration body size in the rubyspot damselfly Hetaerina americana. Evolution 50: 1939-1948. 
Hall DW, Kirkpatrick M, West B (2000). Runaway sexual selection when female preferences are directly selected. Evolution 54: 1862-1869.

Hansen TF (1997). Stabilizing selection and the comparative analysis of adaptation. Evolution 51: 1341-1351.

Hill GE (1998). An easy, inexpensive means to quantify plumage colouration. J Field Ornithol 69: 353-363.

Hoekstra HE, Hoekstra JM, Berrigan D, Vignieri SN, Hoang A, Hill CE et al. (2001). Strength and tempo of directional selection in the wild. Proc Natl Acad Sci USA 98: 9157-9160.

Houle D (1991). Genetic covariance of fitness correlates: what genetic correlations are made of and why it matters. Evolution 45: 630-648.

Hughes JM, Mather PB, Sheldon AL, Allendorf FW (1999). Genetic structure of the stonefly Yoraperla brevis, populations: the extent of gene flow among adjacent montane streams. Freshwater Biol 41: 63-72.

Irwin DE, Bensch S, Price TD (2001). Speciation in a ring. Nature 409: 333-337.

Iwasa Y, Pomiankowski A (1995). Continual change in mate preferences. Nature 377: 420-422.

Koenig WD, Albano SS (1987). Lifetime reproductive success, selection, and the opportunity for selection in the whitetailed skimmer Plathemis lydia (Odonata: Libellulidae). Evolution 41: 22-36.

Laird PW, Zijderveld A, Linders K, Rudnicki MA, Jaenisch R, Berns A (1991). Simplified mammalian DNA isolation procedure. Nucl Acids Res 19: 4293.

Lande R (1980). Genetic variation and phenotypic evolution during allopatric speciation. Am Nat 116: 463-479.

Lande R (1981). Models of speciation by sexual selection on polygenic traits. Proc Natl Acad Sci USA 78: 3721-3725.

Lande R (1982). Rapid origin of sexual isolation and character divergence in a cline. Evolution 36: 213-223.

Lande R (2000). Quantitative genetics and phenotypic evolution. In: Singh RS, Krimbas CB (eds) Evolutionary Genetics: From Molecules to Morphology, Cambridge University Press: Cambridge, UK. pp 335-350.

Lande R, Arnold SJ (1983). The measurement of selection on correlated characters. Evolution 37: 1210-1226.

Lewontin RC (1984). Detecting population frequencies in quantitative characters as opposed to gene frequencies. Am Nat 123: 115-124.

Merilä J (1997). Quantitative trait and allozyme divergence in the Greenfinch (Carduelis chloris, Aves: Fringillidae). Biol J Linn Soc 61: 243-266.

Merilä J, Crnokrak P (2001). Comparisons of genetic differentiation at marker loci and quantitative traits. J Evol Biol 14: 892-903.

Miller MP, Blinn DW, Keim P (2002). Correlations between observed dispersal capabilities and patterns of genetic differentiation in populations of four aquatic insect species from the Arizona White Mountains, USA. Freshwater Biol 47: 1660-1673.

Mitchell-Olds T, Shaw RG (1987). Regression analysis of natural selection: statistical inference and biological interpretation. Evolution 41: 1149-1161.

Panhuis TM, Butlin R, Zuk M, Tregenza T (2001). Sexual selection and speciation. Trends Ecol Evol 16: 364-371.

Payne RJH, Krakauer DC (1997). Sexual selection, space and speciation. Evolution 51: 1-9.

Plaistow SJ, Siva-Jothy MT (1996). Energetic constraints and male mate-securing tactics in the damselfly Calopteryx splendens xanthostoma. Behav Ecol Sociobiol 263: 1233-1238.
Plaistow SJ, Tsubaki Y (2000). A selective trade-off for territoriality and non-territoriality in the polymorphic damselfly Mnais costalis. Proc R Soc London B 267: 969-975.

Price T, Turelli M, Slatkin M (1993). Peak shifts produced by correlated response to selection. Evolution 47: 280-290.

Rantala MJ, Koskimäki J, Taskinen J, Tynkkynen K, Suhonen J (2000). Immunocompetence, developmental stability and wingspot size in the damselfly Calopteryx splendens. Proc $R$ Soc London B 267: 2453-2457.

Raymond M, Rousset F (1995). Genepop (version 2.2): population genetics software for exact tests and ecumenicism. J Hered 86: 248-249.

Rice WR, Holland B (1997). The enemies within: intergenomic conflict, interlocus contest evolution (ICE), and the intraspecific Red Queen. Behav Ecol Sociobiol 41: 1-10.

Rice WR, Hostert E (1993). Laboratory experiments on speciation: what have we learned in 40 years? Evolution 47: 1637-1653.

Rogers AR (1986). Population differences in quantitative characters as opposed to gene frequencies. Am Nat 127: 729-730.

Sandhall $\AA$ (2000). Trollsländor $i$ Europa 2nd edn. Interpublishing: Stockholm, Sweden (in Swedish).

Schluter D (2000). The Ecology of Adaptive Radiation, Oxford University Press, Oxord, U.K.

Schultheis AS, Weigt LA, Hendricks AC (2002). Gene flow, dispersal, and nested clade analysis among populations of the stonefly Peltoperla tarteri in the southern Appalachians. Mol Ecol 11: 317-327.

Simon JL (2000). Resampling Stats Software and User's Guide 1973-2000, Resampling Stats: Arlington, VA.

Siva-Jothy M (1999). Male wing pigmentation may affect reproductive success via female choice in a Calopterygid damselfly (Zygoptera). Behaviour 136: 1365-1377.

Siva-Jothy M (2000). A mechanistic link between parasite resistance and expression of a sexually selected trait in a damselfly. Proc $R$ Soc London B 267: 2523-2527.

Slatkin M (1985). Gene flow in natural populations. Ann Rev Ecol Syst 16: 393-430.

Statsoft (2000). STATISTICA for Windows, Statsoft Inc.: Tulsa, OK

Tilley SG, Verrell PA, Arnold SJ (1990). Correspondence between sexual isolation and allozyme differentiation: a test in the salamander Desmognathus ochrophaeus. Proc Natl Acad Sci USA 87: 2715-2719.

Tregenza T, Pritchard VL, Butlin RK (2000). Patterns of trait divergence between populations of the meadow grasshopper, Chortippus parallellus. Evolution 54: 574-585.

Uy JAC, Borgia G (2000). Sexual selection drives rapid divergence in bowerbird display. Evolution 54: 273-278.

Vos P, Hogers R, Bleeker M, Reijans M, van der Lee T, Hornes M et al (1995). AFLP - a new technique for DNA fingerprinting. Nucl Acids Res 23: 4407-4414.

Waage JK (1979). Reproductive character displacement in Calopteryx (Odonata: Calopterygiidae). Evolution 33: 104-116.

Wade MJ, Goodnight CJ (1998). Perspective: the theories of Fisher and Wright in the context of metapopulations: when nature does many small experiments. Evolution 52: 1537-1553.

Wade MJ, Kalisz SM (1990). The causes of natural selection. Evolution 44: 1947-1955.

West-Eberhard MJ (1983). Sexual selection, social competition and speciation. Q Rev Biol 58: 155-183.

Wright S (1943). Isolation by distance. Genetics 28: 114-138.

Supplementary Information accompanies the paper on Heredity website (http://www.nature.com/hdy). 